\title{
Could the clinical target volume be omitted for radiotherapy of locally advanced non-small cell lung cancer in the modern era?
}

\author{
Vivek Verma, Joe Y. Chang \\ Department of Radiation Oncology, University of Texas M.D. Anderson Cancer Center, Houston, TX, USA \\ Correspondence to: Joe Y. Chang, MD, PhD. Department of Radiation Oncology, Unit 97, University of Texas M.D. Anderson Cancer Center, 1515 \\ Holcombe Blvd, Houston, TX 77030, USA. Email: jychang@mdanderson.org. \\ Comment on: Zou L, Chu L, Xia F, et al. Is clinical target volume necessary? - a failure pattern analysis in patients with locally advanced non-small cell \\ lung cancer treated with concurrent chemoradiotherapy using intensity-modulated radiotherapy technique. Transl Lung Cancer Res 2020;9:1986-95.
}

Submitted Oct 27, 2020. Accepted for publication Nov 19, 2020.

doi: $10.21037 /$ tlcr-2020-19

View this article at: http://dx.doi.org/10.21037/tlcr-2020-19

Radiation therapy (RT) is considered an integral aspect of the definitive management of locally advanced non-small cell lung cancer (LA-NSCLC). Unfortunately, despite the addition of chemotherapy to RT, a substantial proportion of patients suffer local and/or regional recurrence, along with an even greater risk of developing distant metastasis (DM) (1). In the most modern era, the addition of adjuvant durvalumab (2) has substantially altered relapse patterns and prognosis, making it necessary to question whether the historical principles and techniques of RT still apply to this era.

RT target delineation for LA-NSCLC generally proceeds according to the International Commission on Radiation Units and Measurements (ICRU) (3), in which the gross tumor volume (GTV) is initially delineated based on pre-treatment imaging. Expansion of the GTV yields the clinical target volume (CTV), which is then modified based on the proximity to anatomic planes as well as the clinically-judged locations of suspected subclinical disease involvement. An additional expansion, aimed to address variabilities/uncertainties in daily patient positioning and setup, is then added to construct the planning target volume (PTV). The dose of RT is prescribed such that it covers nearly all (commonly, 90-100\%) of the PTV, as long as dose limits to nearby organs-at-risk (OARs) are not exceeded.

One potential way to reduce the OAR dose exposure is to reduce the irradiated volume (i.e., the PTV). This is most commonly performed by reducing the expansion that transforms the CTV into the PTV. Doing so has been made possible in the modern era, as high-quality volumetric image guidance as well as respiratory motion management techniques lead to high credence that the tumor does not fall outside the target volumes. As a result, most practicing clinicians utilize smaller PTV expansions than the past, an element that has generally reduced toxicities as compared to the prior era.

A study from the Shanghai Cancer Center (4) represents a novel approach to reducing the irradiated volume by means of omitting the CTV. This novel concept is intriguing in the modern era, and must be understood in the overall context of the purpose of creating a CTV. The expansion from GTV to CTV can occur in two primary manners: (I) a predetermined margin to cover the areas immediately adjacent to grossly visible disease, and/or (II) areas that are electively irradiated due to a high risk of subclinical involvement. It is true for nearly every irradiated neoplasm that microscopic disease always requires a dose lower than that of gross disease. However, in the radiotherapeutic management of LA-NSCLC, the areas at risk for subclinical involvement (i.e., the CTV) is prescribed the same dose as the gross disease (i.e., the GTV), which is not intuitively logical. To this extent, altering this historical practice is a much-needed "thought experiment" that could reduce toxicities in this population and improve quality of life.

The first rationale for potentially omitting the CTV in LA-NSCLC is a historical precedent. Elective nodal RT in LA-NSCLC was the standard of care before the mid-late 2000s, when studies demonstrated that recurrence in these elective areas occurred infrequently (5-7), and removing these electively treated areas reduced toxicities. These findings are likely explained by the fact that the patterns of 
failure in these patients are dominated by DM and in-field failure. However, in the contemporary era, when patients are surviving longer and both in-field and distant relapses are considerably lower, evaluating the safety and efficacy of CTV-omitted RT certainly merits scientific and clinical study.

Moreover, CTV omission is standard practice for all early-stage NSCLC cases treated with stereotactic ablative RT (SABR). Most commonly, gross disease is outlined at given points of the respiratory cycle and is then uniformly expanded directly into a PTV; the areas of subclinical involvement immediately surrounding the GTV are intentionally not covered (although they are often encompassed within the PTV). The rate of failure immediately surrounding the PTV (so-called "marginal miss") is very uncommon with SABR, and predictably so, because the dominant relapse pattern after SABR is DM (similar to the case of LA-NSCLC). Importantly, the use of contemporary high-quality image guidance has allowed for these small target expansions in the SABR setting, and it is postulated that doing the same for LA-NSCLC cases is essential if CTV-omitted RT for LA-NSCLC is to advance in clinical study and practice.

An additional rationale for this novel approach is that modern diagnostic workup is far superior to that of the past, implying that subclinical disease can be more adequately localized as compared to the past. For instance, uniform use of positron emission tomography (PET) assists in prudent target delineation for many neoplasms (8), and performing pathologic mediastinal nodal staging (by means of endobronchial ultrasound, endoscopic ultrasound, and/or mediastinoscopy) may offer a greater confidence that only the truly involved areas are being irradiated.

The main finding of Zou et al. was that the primary mode of recurrence in their population was DM, and to a lesser degree local failure; recurrence (especially isolated recurrence) in/near the omitted CTV area was quite rare. However, most of their population was treated prior to durvalumab becoming the standard of care for LA-NSCLC. As a result, it is imperative to evaluate how alterations in patterns of failure after durvalumab affect whether the CTV can be safely omitted or not. A patterns of failure analysis of the PACIFIC trial was presented in abstract form at the 2019 Annual Meeting of the American Society for Radiation Oncology (9). This study showed that $45.4 \%$ of patients in the durvalumab arm had experienced progression: intrathoracic progression in $36.6 \%$, extrathoracic progression in $6.9 \%$, and both (simultaneous) in $3.4 \%$.
These results suggest that improved systemic control from durvalumab has shifted the patterns of failure, and as a result, intrathoracic control has become substantially more important. To this extent, continued caution in omitting the CTV from LA-NSCLC is encouraged until prospective data corroborate the retrospective findings of Zou and coworkers. Although the PACIFIC secondary analysis did not further subdivide intrathoracic progression into in-field and out-of-field relapse, this distinction is very important because it has clear implications on the potential for further exploration of CTV-omitted RT for LA-NSCLC in the context of durvalumab.

Although Zou and colleagues did not report toxicity outcomes of their approach, they importantly showed that reducing treatment volumes better spares lymphocytes, an essential component of the immune system by which immunotherapy compounds exert anti-neoplastic effects. Recent research has shown that lymphopenia (and RTinduced lymphopenia) is associated with lower likelihood of immune-mediated systemic effects and poorer prognosis in lung cancer patients receiving combined immunotherapy and RT (10-12). Because lymphocytes are the "effector cells" of immune checkpoint inhibitors, reducing lymphopenia should be a prime goal of reduced-field RT in the durvalumab era; however, as mentioned above, this should be carefully weighed against the potential risk of recurrences by omitting the CTV.

Altogether, the investigation (4) is an excellent example of how the clear benefits of durvalumab for LA-NSCLC are requiring a revision in the thought processes for predurvalumab chemoradiation. For instance, improved systemic control from durvalumab and the resulting shift in patterns of failure may require revisitation of the role of dose-escalation using safe techniques such as intensitymodulated RT (IMRT). Although RTOG 0617 did not determine any benefits to doing so (1), the propensity for developing DM in the non-immunotherapy setting likely dampened any potential benefits of improved local control. The use of IMRT in only around one-half of patients also limits conclusions regarding dose-escalation versus safe dose-escalation.

In the era of immunotherapy and targeted therapy, we should consider routinely conducting molecular profiling (including PD-L1 expression testing) and reexplore the strategy of induction systemic therapy for LA-NSCLC. Doing so may not only reduce DM further (owing to the earlier delivery of systemic therapy), but also better evaluate tumor biology and allow for risk- 
adapted RT management, particularly for patients with extensive involvement and/or bulky disease. For example, if up-front immunotherapy/targeted therapy causes a good response, then the reduction in disease bulk would naturally result in reduced treatment volumes and lower RT-induced toxicities/lymphopenia. In patients with favorable tumor biology in whom clinicians may be more confident that subclinical disease is indeed eliminated, the benefit of CTV-omitted RT may be proportionally higher. Conversely, patients who have stable or progressive disease may require dose-escalated $\mathrm{RT}$ and/or non-CTVomitted RT.

Taken together, reducing the irradiated volume is an important consideration to improving the therapeutic ratio for LA-NSCLC patients. Whereas technological improvements in RT targeting and delivery have led to a reduction in PTV expansions as compared to the past, the novel approach of omitting the CTV represents an intriguing new method to continually attempt to further enhance the therapeutic ratio. The notion propagated by Zou and colleagues is in its infancy, however, and prospective observational studies are required. Until these trials are published, it may be worth-at minimumimplementing the use of more prudent CTV expansions and/or avoiding to prescribe the same dose to subclinical areas as is delivered to gross disease, especially in the era of technologically-advanced RT and immunotherapy.

\section{Acknowledgments}

Funding: None.

\section{Footnote}

Provenance and Peer Review: This article was commissioned by the Guest editors (Steven H. Lin, Xiaolong Fu and Zhengfei Zhu) for the series "New era of treatment for unresectable locally advanced non-small cell lung cancer" published in Translational Lung Cancer Research. The article did not undergo external peer review.

Conflicts of Interest: Both authors have completed the ICMJE uniform disclosure form (available at http:// dx.doi.org/10.21037/tlcr-2020-19). The series "New era of treatment for unresectable locally advanced nonsmall cell lung cancer" was commissioned by the editorial office without any funding or sponsorship. JYC has received grant from BMS-MDACC, personal fees from
AstraZeneca and Varian, he is a shareholder of Global Oncology One, has served on the advisory board for Legion, outside the submitted work. VV has no conflicts of interest to declare.

Ethical Statement: The authors are accountable for all aspects of the work in ensuring that questions related to the accuracy or integrity of any part of the work are appropriately investigated and resolved.

Open Access Statement: This is an Open Access article distributed in accordance with the Creative Commons Attribution-NonCommercial-NoDerivs 4.0 International License (CC BY-NC-ND 4.0), which permits the noncommercial replication and distribution of the article with the strict proviso that no changes or edits are made and the original work is properly cited (including links to both the formal publication through the relevant DOI and the license). See: https://creativecommons.org/licenses/by-nc-nd/4.0/.

\section{References}

1. Bradley JD, Hu C, Komaki RR, et al. Long-term results of NRG Oncology RTOG 0617: Standard- versus highdose chemoradiotherapy with or without cetuximab for unresectable stage III non-small-cell lung cancer. J Clin Oncol 2020;38:706-14.

2. Antonia SJ, Villegas A, Daniel D, et al. Overall survival with durvalumab after chemoradiotherapy in stage III NSCLC. N Engl J Med 2018;379:2342-50.

3. ICRU. Prescribing, Recording and Reporting Photon Beam Therapy. Report 50. Bethesda, MD: International Commission on Radiation Units and Measurements, 1999.

4. Zou L, Chu L, Xia F, et al. Is clinical target volume necessary? - a failure pattern analysis in patients with locally advanced non-small cell lung cancer treated with concurrent chemoradiotherapy using intensity-modulated radiotherapy technique. Transl Lung Cancer Res . 2020;9:1986-95.

5. Yuan S, Sun X, Li M, et al. A randomized study of involved-field irradiation versus elective nodal irradiation in combination with concurrent chemotherapy for inoperable stage III nonsmall cell lung cancer. Am J Clin Oncol 2007;30:239-44.

6. Rosenzweig KE, Sura S, Jackson A, et al. Involved-field radiation therapy for inoperable non small-cell lung cancer. J Clin Oncol 2007;25:5557-61.

7. Sulman EP, Komaki R, Klopp AH, et al. Exclusion of 
elective nodal irradiation is associated with minimal elective nodal failure in non-small cell lung cancer. Radiat Oncol 2009;4:5.

8. Verma V, Choi JI, Sawant A, et al. Use of PET and Other Functional Imaging to Guide Target Delineation in Radiation Oncology. Semin Radiat Oncol 2018;28:171-7.

9. Raben A, Rimner A, Senan S, et al. Patterns of Disease Progression with Durvalumab in Stage III Non-small Cell Lung Cancer (PACIFIC). Int J Radiat Oncol Biol Phys 2019; 105:LBA6.

10. Chen D, Verma V, Patel RR, et al. Absolute Lymphocyte Count Predicts Abscopal Responses and Outcomes

Cite this article as: Verma V, Chang JY. Could the clinical target volume be omitted for radiotherapy of locally advanced non-small cell lung cancer in the modern era? Transl Lung Cancer Res 2021;10(1):5-8. doi: 10.21037/tlcr-2020-19 in Patients Receiving Combined Immunotherapy and Radiation Therapy: Analysis of 3 Phase 1/2 Trials. Int J Radiat Oncol Biol Phys 2020;108:196-203.

11. Chen D, Patel RR, Verma V, et al. Interaction between lymphopenia, radiotherapy technique, dosimetry, and survival outcomes in lung cancer patients receiving combined immunotherapy and radiotherapy. Radiother Oncol 2020;150:114-20.

12. Patel RR, Verma V, Barsoumian H, et al. Use of MultiSite Radiation Therapy as Systemic Therapy: A New Treatment Approach Personalized by Patient Immune Status. Int J Radiat Oncol Biol Phys 2021;109:352-64. 